Pacific Journal of Mathematics

NORMAL STRUCTURE AND THE SUM-PROPERTY 


\title{
NORMAL STRUCTURE AND THE SUM-PROPERTY
}

\author{
Thomas R. LANDES
}

For $Z=\left(\mathbf{R}^{N},|\cdot|\right)$ with symmetric norm $|\cdot|$ the $Z$-direct sum of the normed spaces $X_{1}, \ldots, X_{N}$ is its product space with norm $\left\|\left(x_{1}, \ldots, x_{N}\right)\right\|$ $=\left|\left(\left\|x_{1}\right\|, \ldots,\left\|x_{N}\right\|\right)\right|$. A normed space $X$ is said to have the sum-property (SP) if each $Z$-direct sum of finitely many copies of $X$ has normal structure (NS). It turns out that the class of spaces having the SP is the largest subclass of the class of spaces having NS which is closed under each finite Z-direct sum operation. The SP is characterized by the property that limit-affine (i.e., the functional $\Lambda(x)=\lim \left\|x_{n}-x\right\|$ is defined and affine on $\left.\operatorname{conv}\left(\left\{x_{n}\right\}\right)\right)$ sequences $\left\{x_{n}\right\}$ with non-decreasing $\left\{\Lambda\left(x_{n}\right)\right\}$ are constant.

In contrast to a previous conjecture it is shown that every infinite dimensional separable normed space can be renormed to have NS and not the SP. Moreover, in order that NS is inherited from $X_{1}, \ldots, X_{N}$ to its Z-direct sum, it is not only sufficient (as previously shown) but also necessary that each line segment (if there is any) in the unit sphere of $Z$ lies in a hyperplane $\left\{z \mid z_{l}=\alpha\right\}$ for some $i \leq N, \alpha \neq 0$. In fact, if $Z$ does not satisfy this condition, and if infinite dimensional separable normed spaces $X_{1}, \ldots, X_{N}$ are given, then there are normed spaces $Y_{l}$ with NS isomorphic to $X_{i}$ whose $Z$-direct sum does not have NS.

Finally, it is shown that a normed space with a symmetric (not necessarily countable) basis can be renormed to have NS if and only if it can be renormed to be uniformly convex in every direction. In particular, $c_{0}(I)$ can be renormed to have NS if and only if $I$ is countable. As a counter-example, a reflexive normed space with an unconditional basis is given which has the SP but cannot be renormed to be uniformly convex in every direction. All results hold also for weakly NS and the weak SP.

Introduction. A subset $A$ of a normed space $X$ is said to have normal structure (NS for short) if every bounded convex subset $C$ of $A$ with positive diameter $d=\sup \{\|x-y\| x, y \in C\}$ is contained in some ball with center in $C$ and radius strictly smaller than $d$. This property, introduced by Brodskii and Mil'man [1], is important in fixed point theory for nonexpansive mappings. We refer the interested reader to [3], [4], [5], [6], [8].

In applications, also weakly normal structure (WNS for short) is of interest. A normed space $X$ is said to have weakly normal structure if every weakly compact subset of $X$ has NS.

In [10], the author has analyzed stability properties of (W)NS. (The term (W)NS means both NS and WNS, the "W" refers to all expressions occurring in brackets. For example, "the (weak) SP implies (W)NS" 
means both "the SP implies NS" and "the weak SP implies WNS".) The following problem was raised in [10]:

\section{P1: Is (W)NS preserved under every finite direct-sum-operation?}

An affirmative answer to P1 would mean that, given any symmetric norm $|\cdot|$ on $\mathbf{R}^{N}$ - i.e., $\left|\left(\xi_{1}, \ldots, \xi_{N}\right)\right|=\left|\left(\left|\xi_{1}\right|, \ldots,\left|\xi_{N}\right|\right)\right|$ - and given arbitrary normed spaces $X_{1}, \ldots, X_{N}$ with (W)NS, the direct sum $X_{1}$ $\oplus \cdots \oplus X_{N}$ has (W)NS with respect to the norm $\left\|\left(x_{t}\right)_{i=1}^{N}\right\|=\left|\left(\left\|x_{i}\right\|_{i}\right)_{i=1}^{N}\right|$; this direct sum is written as $\left(\sum_{i=1}^{N} \oplus X_{i}\right)_{Z}, Z=\left(\mathbf{R}^{N},|\cdot|\right)$, and called the $Z$-direct sum of $X_{1}, \ldots, X_{N}$. In [10], it is shown that (W)NS is preserved under such a $Z$-direct-sum-operation whenever each - if there is any - line segment belonging to the positive part of the unit sphere of $Z$ is contained in a hyperplane not containing 0 orthogonal to some coordinate axis. This latter condition is formally written in the following way (condition $(*)$ in $[\mathbf{1 0}])$ :

$$
\begin{aligned}
& \text { If }\left|\left(\xi_{i}\right)_{i=1}^{N}\right|=\left|\left(\eta_{l}\right)_{i=1}^{N}\right|=\left|\frac{1}{2}\left(\xi_{i}+\eta_{l}\right)_{i=1}^{N}\right|=1 \text { and } \xi_{l} \geq 0, \eta_{i} \geq 0 \\
& \text { for all } i=1, \ldots, N \text {, then } \xi_{l}=\eta_{i}>0 \text { for some } i \leq N
\end{aligned}
$$

All $l_{p}^{N}$-norms, $1<p \leq \infty$, satisfy (SC), all strictly convex norms on $\mathbf{R}^{N}$ satisfy (SC), but not the $l_{1}^{N}$-norm. We show in $\S 4$ that (SC) is not only sufficient but also necessary for preservation of (W)NS. Consequently, P1 is solved in the negative.

In [10], the isomorphic analogue of (W)NS is also dealt with: A normed space $X$ is said to have (weakly) isonormal structure - (W)INS for short - if $X$ is isomorphic to a normed space with (W)NS. In [10], it is shown that $X$ has (W)INS if and only if there is a bounded linear 1-1 operator $T$ from $X$ into a normed space with (W)NS. Especially, every separable normed space has INS:

$$
T x:=\left(2^{-n}\left\langle x_{n}^{*}, x\right\rangle\right) \in l_{2} \quad \text { if }\|x\|=\sup _{n \in \mathbf{N}}\left|\left\langle x_{n}^{*}, x\right\rangle\right| \text { for all } x \in X .
$$

Moreover, given any normed space $X$, the subclass of $\operatorname{ISO}(X)$ - the class of all spaces isomorphic to $X$ - of all spaces with (W)NS is either empty or dense in $\operatorname{ISO}(X)$ in the topology induced by the Banach-Mazur distance.

The following question was left open in [10]:

P2. Does $c_{0}(I)$ have (W)INS for uncountable I?

In $\S 5$, we give the negative answer: $c_{0}(I)$ has (W)INS if and only if $I$ is countable, i.e., if and only if $c_{0}(I)$ can be renormed to be uniformly 
convex in every direction. This latter characterization extends to the class of spaces with a symmetric basis but not to the class of spaces with an unconditional basis.

2. Limit-affine sequences. Problem $P 1$ leads in a natural way to the consideration of limit-affine sequences as we will see.

Given a bounded sequence $\left\{x_{n}\right\}$ in a normed space $X$, we consider the limit-functional

$$
\Lambda(x)=\lim _{n \rightarrow \infty}\left\|x_{n}-x\right\|
$$

being defined for all $x \in X$ for which the right hand side limit exists. The sequence $\left\{x_{n}\right\}$ is then called limit-affine if its limit-functional is defined on the whole convex hull of $\left\{x_{n}\right\}$ and is affine there. If a growth condition such as "increasingly", "non-increasingly", "constantly" etc. is added to the term "limit-affine", then this indicates that the sequence $\left\{\Lambda\left(x_{n}\right)\right\}$ has this growth behaviour; for example, an increasingly limit-affine sequence $\left\{x_{n}\right\}$ is a limit-affine sequence for which $\left\{\Lambda\left(x_{n}\right)\right\}$ is increasing.

A constantly limit-affine sequence is called limit-constant. The associated limit-functional is constant on the convex hull of a limit-constant sequence.

A non-decreasingly (non-increasingly, respectively) limit-affine sequence $\left\{x_{n}\right\}$ is either constant (Cauchy, resp.) or has no Cauchy subsequence and the former is the case if and only if $\Lambda\left(x_{n}\right) \equiv 0\left(\Lambda\left(x_{n}\right) \rightarrow 0\right.$, resp.).

Limit-constant sequences arise in connection with (W)NS:

Proposition 1 ([10]). The normed space $X$ has (W)NS if and only if every (weakly convergent) limit-constant sequence in $X$ is constant.

For the solution of $\mathrm{P} 1$, one especially has to consider the $l_{1}^{2}$-norm, that is, one has to solve:

P1'. Does $X \oplus_{1} Y$, the space $X \oplus Y$ with norm $\|(x, y)\|=\|x\|+\|y\|$, have (W)NS if both $X$ and $Y$ have (W)NS?

If $\left\{\left(x_{n}, y_{n}\right)\right\}$ is limit-constant in $X \oplus_{1} Y$, then both $\left\{x_{n}\right\}$ and $\left\{y_{n}\right\}$ are limit-affine and, passing to some subsequence, either both are limit-constant or one is increasingly limit-affine and the other decreasingly limitaffine. This demonstrates what we meant above with "...P1 leads ... to ... limit-affine sequences...". 
Limit-affineness together with its growth condition inherits to subsequences and is preserved under the operation $x_{n} \rightarrow \alpha x_{n}+x, \alpha \neq 0$, $x \in X$. So, if there is a limit-affine sequence $\left\{x_{n}\right\}$ in $X$ with $\inf _{n \in \mathbf{N}} \Lambda\left(x_{n}\right)>0$ (i.e., $\left\{x_{n}\right\}$ has no Cauchy subsequence), then we may assume that $\left\{x_{n}\right\}$ is increasingly or constantly or decreasingly limit-affine with $\Lambda\left(x_{n}\right) \rightarrow 1$. Moreover, if some subsequence of $\left\{x_{n}\right\}$ converges in some vector space topology on $X$, then we may assume that $\left\{x_{n}\right\}$ converges to 0 in this topology. If this topology is the weak topology, then $\Lambda(0)=\lim _{n \rightarrow \infty}\left\|x_{n}\right\|=1$. (See $[\mathbf{1 0}]$.)

Finally, every limit-affine sequence has a subsequence all of whose subsequences $\left\{x_{n}\right\}$ satisfy:

$$
\text { (DS) } \lim _{n \rightarrow \infty}\left\{\sum_{k=1}^{n}\left\|x_{n+1}-x_{k}\right\|-n\left\|x_{n+1}-\bar{x}_{n}\right\|\right\}=0, \quad \bar{x}_{n}=\frac{1}{n} \sum_{k=1}^{n} x_{k} \text {. }
$$

Vice versa, if $\left\{x_{n}\right\}$ satisfies (DS), then it has a limit-affine subsequence (cf. [10]).

Proposition 2. Let $X$ be a normed space and $Y \subset X^{*}$ such that

$$
\|x\|=\sup \{\mid\langle y, x\rangle \| y \in Y\} \quad \text { for all } x \in X .
$$

Let $\left\{x_{n}\right\} \subset X$ be limit-affine and $\Lambda_{\infty}$ be a cluster-point of $\left\{\Lambda\left(x_{n}\right)\right\}$. Then, there is a sequence $\left\{y_{n}\right\}$ in $Y$ and an increasing sequence $\left\{m_{n}\right\}$ such that

(i) $\left\{\Lambda\left(x_{m_{n}}\right)\right\}$ is monotonous with limit $\Lambda_{\infty}$.

(ii) $\alpha=\lim _{n \rightarrow \infty}\left\langle y_{n}, x_{m_{n}}\right\rangle$ exists.

(iii) There is $a \beta \in \mathbf{C}$ with $|\beta|=1$ such that

$$
\lim _{n \rightarrow \infty}\left\langle y_{n}, x\right\rangle=\alpha-\Lambda(x) \beta \quad \text { for all } x \in \overline{\operatorname{conv}}\left(\left\{x_{n}\right\}\right) .
$$

(iv) $|\alpha-\Lambda(x) \beta| \leq\|x\|$ for all $x \in \overline{\operatorname{conv}}\left(\left\{x_{n}\right\}\right)$.

(v) If $\Lambda_{\infty}>0$, then $\left\{y_{n}\right\}$ has no constant subsequence.

If , additionally, $x_{n} \rightarrow 0$ weakly, then

(vi) $\Lambda_{\infty}=\lim _{n \rightarrow \infty} \Lambda\left(x_{n}\right)=\Lambda(0)$ and $\alpha=\Lambda_{\infty} \beta$.

If $Y$ vanishes pointwise at infinity, i.e., if for all $x \in X$ and $\varepsilon>0$ there is $a$ finite $F \subset Y$ such that $|\langle y, x\rangle|<\varepsilon$ for all $y \in Y \backslash F$, then

(vii) $\left\{x_{n}\right\}$ is either Cauchy or limit-constant with $\alpha=\Lambda_{\infty} \beta$.

Proof. Put $\Lambda_{k}=\Lambda\left(x_{k}\right)$. Choose $\left\{p_{n}\right\}$ increasing such that $\Lambda_{p_{n}} \rightarrow \Lambda_{\infty}$, $\left\|x_{p_{n}}-x_{k}\right\|<\Lambda_{k}+n^{-2}, k \leq n$, and $\left\|x_{p_{n}}-\bar{x}_{n}\right\|>\Lambda\left(\bar{x}_{n}\right)-n^{-2}$. Then

$$
0 \leq \Delta_{n}=\sum_{k=1}^{n}\left\|x_{p_{n}}-x_{k}\right\|-n\left\|x_{p_{n}}-\bar{x}_{n}\right\|<\frac{2}{n} \rightarrow 0 .
$$


Choose $\tilde{y}_{n} \in Y$ such that $\left\langle\tilde{y}_{n}, x_{p_{n}}-\bar{x}_{n}\right\rangle \mid>\left\|x_{p_{n}}-\bar{x}_{n}\right\|-n^{-2}$. Fix an arbitrary $x=\sum_{k=1}^{m} \lambda_{k} x_{k} \in \operatorname{conv}\left(\left\{x_{n}\right\}\right)$ and put $\lambda_{k}=0, k>m$. Since $n\left(x_{p_{n}}-\bar{x}_{n}\right)=x_{p_{n}}-x+\sum_{k=1}^{n}\left(1-\lambda_{k}\right)\left(x_{p_{n}}-x_{k}\right)$ we obtain

$$
\begin{aligned}
& n\left\|x_{p_{n}}-\bar{x}_{n}\right\|-\frac{1}{n}<n\left|\left\langle\tilde{y}_{n}, x_{p_{n}}-\bar{x}_{n}\right\rangle\right| \\
& \quad \leq\left|\left\langle\tilde{y}_{n}, x_{p_{n}}-x\right\rangle\right|+\sum_{k=1}^{n}\left(1-\lambda_{k}\right)\left|\left\langle\tilde{y}_{n}, x_{p_{n}}-x_{k}\right\rangle\right|
\end{aligned}
$$

and

$$
\begin{aligned}
\Lambda(x) & \leftarrow\left\|x_{p_{n}}-x\right\| \geq\left|\left\langle\tilde{y}_{n}, x_{p_{n}}-x\right\rangle\right| \\
& \geq n\left|\left\langle\tilde{y}_{n}, x_{p_{n}}-\bar{x}_{n}\right\rangle\right|-\sum_{k=1}^{n}\left(1-\lambda_{k}\right)\left|\left\langle\tilde{y}_{n}, x_{p_{n}}-x_{k}\right\rangle\right| \\
& >n\left\|x_{p_{n}}-\bar{x}_{n}\right\|-\frac{1}{n}-\sum_{k=1}^{n}\left(1-\lambda_{k}\right)\left\|x_{p_{n}}-x_{k}\right\| \\
& =\sum_{k=1}^{m} \lambda_{k}\left\|x_{p_{n}}-x_{k}\right\|-\Delta_{n}-\frac{1}{n} \rightarrow \sum_{k=1}^{m} \lambda_{k} \Lambda_{k}=\Lambda(x)
\end{aligned}
$$

and we have

$$
\left|\left\langle\tilde{y}_{n}, x_{p_{n}}-x\right\rangle\right| \rightarrow \Lambda(x) \text { for all } x \in \operatorname{conv}\left(\left\{x_{n}\right\}\right) .
$$

Choose an increasing sequence $\left\{k_{n}\right\}$ such that (i) and (ii) hold for $y_{n}=\tilde{y}_{k_{n}}$ and $m_{n}=p_{k_{n}}$ and that $\beta_{k}=\lim _{n \rightarrow \infty}\left\langle y_{n}, x_{k}\right\rangle$ exists for each $k$. Formula $(*)$ yields $\left|\alpha-\beta_{k}\right|=\Lambda_{k}$ and

$$
\Lambda_{k}+\Lambda_{1}=\left|2 \alpha-\beta_{k}-\beta_{1}\right| \leq\left|\alpha-\beta_{k}\right|+\left|\alpha-\beta_{1}\right|=\Lambda_{k}+\Lambda_{1} .
$$

Since $|\cdot|$ on $\mathbf{C}$ is strictly convex, $\alpha-\beta_{k}=\Lambda_{k} \beta$ for some $\beta \in \mathbf{C}$ with $|\beta|=1$. A continuity argument implies (iii), and (iv) is an immediate consequence of (iii).

If some subsequence $\left\{y_{r_{n}}\right\}$ of $\left\{y_{n}\right\}$ is constant and $\Lambda_{\infty}>0$,

$$
\alpha \neq \alpha-\Lambda_{\infty} \beta \underset{k \rightarrow \infty}{\leftarrow} \alpha-\Lambda_{m_{r_{k}}} \beta \underset{n \rightarrow \infty}{\leftarrow}\left\langle y_{r_{n}}, x_{m_{r_{k}}}\right\rangle=\left\langle y_{r_{k}}, x_{m_{r_{k}}}\right\rangle \underset{k \rightarrow \infty}{\rightarrow} \alpha
$$

which is absurd.

If $x_{n} \rightarrow 0$ weakly, then $0 \in \overline{\operatorname{conv}}\left(\left\{x_{n}\right\}\right)$ and (vi) follows from (iv) and [10, Remark 1].

Let $Y$ vanish pointwise at infinity. If $\left\{x_{n}\right\}$ is not Cauchy, then $\left\{\Lambda\left(x_{n}\right)\right\}$ has a cluster-point $\Lambda_{\infty}>0$. Choose $\left\{y_{n}\right\}$ and $\left\{m_{n}\right\}$ with (i) $-(\mathrm{v})$. Then $0 \leftarrow\left\langle y_{n}, x_{k}\right\rangle \rightarrow \alpha-\Lambda_{k} \beta$, which implies that $\left\{\Lambda_{k}\right\}$ is constant, i.e., $\Lambda_{k}=\Lambda_{\infty}$ for all $k$ and $\alpha=\Lambda_{\infty} \beta$. 
COROllaRy 1. Every limit-affine sequence in $c_{0}(I)$ is either limit-constant or convergent.

3. The sum-property. The observations on $\mathrm{P} 1^{\prime}$ mentioned in $\S 2$ motivated the author in [10] to define a property, called the sum-property, in such a way that the "bad" case ( $\left\{x_{n}\right\}$ increasingly, $\left\{y_{n}\right\}$ decreasingly, $\left\{\left(x_{n}, y_{n}\right)\right\}$ constantly limit-affine) is excluded: non-decreasingly limit-affine sequences have to be constant by definition of the sum-property. But, this definition seems to be rather unnatural and the name "sum-property" gives no association for its defining property. So, we proceed here in another way (but come to the same goal). In view of the solution of P1, we could simply define the (weak) sum-property to be the weakest property of normed spaces which is stronger than (i.e., stronger than or equivalent to) (W)NS and is preserved under every finite direct-sum-operation (FDSO for short). This "definition" is somewhat "dangerous" in view of logical loops and cannot be written in the form " $X$ has the (weak) sum-property if ...". These difficulties can be avoided in restricting the direct sums to powers of one space and in requiring these direct sums to have only (W)NS:

Definition 1. The normed space $X$ is said to have the (weak) sum-property ((W)SP for short) if each Z-direct sum of finitely many copies of $X$ has (W)NS, i.e., if $X^{N}$ has (W)NS with respect to the norm $\left\|\left(x_{l}\right)_{i=1}^{N}\right\|=\left|\left(\left\|x_{\imath}\right\|\right)_{i=1}^{N}\right|$ whenever $|\cdot|$ is a symmetric norm on $\mathbf{R}^{N}$.

Obviously, the (W)SP is stronger than (W)NS and weaker than every property of normed spaces which is stronger than (W)NS and preserved under every FDSO. So, the (W)NS is the weakest such property provided it is preserved under every FDSO. This is shown in Theorem 1:

THEOREM 1. The following are equivalent.

(1) $X$ has the (W)SP.

(2) $X \oplus_{1} \mathbf{K}=(X \oplus \mathbf{K})_{l_{1}^{2}}$ has (W)NS, where $\mathbf{K}$ is the scalar field $\mathbf{R}$ or $\mathbf{C}$.

(3) Every non-decreasingly limit-affine (weakly convergent) sequence in $X$ is constant.

Moreover, the (W)SP is the weakest property of normed spaces which is stronger than (W)NS and preserved under every FDSO. 
Proof. (1) $\Rightarrow(2) . X \oplus_{1} \mathbf{K}$ is a subspace of $X \oplus_{1} X$.

$(2) \Rightarrow(3)$. If $\left\{x_{n}\right\}$ is non-decreasingly limit-affine in $X$, then $\left\{x_{n}, \Lambda\left(x_{n}\right)\right\}$ is limit-constant in $X \oplus_{1} \mathbf{K}$.

(3) $\Rightarrow(1)$ and the additional statement. Consequence 3 of [10] states that (3) is preserved under every FDSO.

So, our definition here for the (W)SP coincides with that given in [10]. Moreover, Theorem 1 shows that $\mathrm{P} 1$ and $\mathrm{P} 1{ }^{\prime}$ are equivalent and both equivalent to

\section{$\mathrm{P} 1^{\prime \prime}$. Does (W)NS imply the (W)SP?}

Problem P1" was stated in [10] as a positive conjecture. A priori, there is no reason why (W)SP and (W)NS should be equivalent, although (2) of Theorem 1 suggests that both properties differ not too much. But, if one checks all "classical" sufficient but not necessary conditions for (W)NS (e.g., uniform convexity (in every direction), uniform smoothness, nearly uniform convexity, $k$-uniform rotundity, Opial's condition, condition of Gossez and Lami Dozo,... ) one finds out that all these conditions imply the (W)SP, too (cf. [10]). Moreover, all "classical" sequence spaces have the (W)SP as soon as they have (W)NS: $c_{0}$, all modular sequence spaces including the class of Orlicz sequence spaces as well as $l_{p}, 1 \leq p \leq \infty$ (cf. [11], [12]), all Lorentz sequence spaces $d(w, p)$ (all $d(w, p)$ satisfy Opial's condition so that all $d(w, p)$ have WSP; for $p>1, d(w, p)$ is reflexive, thus, has the SP; $d(w, 1)$ does not have NS because $\left\{\left(\sum_{k=1}^{n} w_{k}\right)^{-1} \sum_{k=1}^{n} e_{k}\right\}$ is limit-constant).

Furthermore, our Corollary 1 implies that every normed space which is isometric to a subspace of $c_{0}(I)$ has the (W)SP as soon as it has (W)NS.

Finally, subspaces of spaces with an "asymptotically co-contractive" basis - i.e., $\liminf \operatorname{in}_{n \rightarrow \infty}\left\|\bar{P}_{n}\right\|=1, \bar{P}_{n}$ the complement of the canonical projection $P_{n}$ - have the WSP if they have WNS. Indeed, if $\left\{x_{n}\right\}$ is non-decreasingly limit-affine, $x_{n} \rightarrow 0$ weakly and $\left\|\bar{P}_{m_{n}}\right\| \rightarrow 1$, then $\Lambda(0)$ $=\lim _{n \rightarrow \infty} \Lambda\left(x_{n}\right) \geq \Lambda\left(x_{1}\right)$ and

$$
\begin{aligned}
& \Lambda\left(x_{1}\right) \underset{\nu \rightarrow \infty}{\leftarrow}\left\|\bar{P}_{m_{\nu}}\right\| \Lambda\left(x_{1}\right) \underset{n \rightarrow \infty}{\leftarrow}\left\|\bar{P}_{m_{\nu}}\right\|\left\|x_{n}-x_{1}\right\| \\
& \quad \geq\left\|\bar{P}_{m_{\nu}}\left(x_{n}-x_{1}\right)\right\| \geq\left\|x_{n}\right\|-\left\|P_{m_{\nu}} x_{n}\right\|-\left\|\bar{P}_{m_{\nu}} x_{1}\right\| \\
& \quad \underset{n \rightarrow \infty}{\rightarrow} \Lambda(0)-\left\|\bar{P}_{m_{\nu}} x_{1}\right\| \underset{\nu \rightarrow \infty}{\rightarrow} \Lambda(0) .
\end{aligned}
$$


So, if there is a universal (containing every separable normed space) space with an asymptotically co-contractive basis, then WSP and WNS are equivalent.

Taking all these arguments into account, one can really hope that $P 1^{\prime \prime}$ has an affirmative solution at least for the weak properties or restricted to the class of reflexive spaces.

4. The solution. The existence of a universal space with an asymptotically co-contractive basis would imply that there is no increasingly limit-affine weakly convergent sequence at all. Such a space cannot exist! Indeed, the sequence $\left\{z_{n}\right\}$ in $l_{\infty}$

$$
z_{n}(j)= \begin{cases}0, & j<n \\ 1-\varepsilon_{n} & j=n, \\ \varepsilon_{n}, & j>n,\end{cases}
$$

is increasingly limit-affine $\left(\Lambda\left(z_{k}\right)=1-\varepsilon_{k}\right)$ and converges weakly to 0 if $\left\{\varepsilon_{n}\right\}$ converges decreasingly to 0 .

Does the subspace of $l_{\infty}$ spanned by $\left\{z_{n}\right\}$ solve $\mathrm{P} 1^{\prime \prime}$ in the negative? No! The sequence $\left\{z_{n+1}-\varepsilon_{n+1} \varepsilon_{n}^{-1} z_{n}\right\}$ is limit-constant. But, some modifications of the above sequence yield a negative solution of $\mathrm{P}^{\prime \prime}$. We even introduce a number of equivalent norms on $c_{0}$ which solve P1" negatively in several ways.

To have easier notation we define the norm \|\| of $X$ to be of type $\tau_{1} \tau_{2} \tau_{3} \tau_{4}, \tau_{i} \in\{0,1\}$, if $(X,\|\|)$ does or does not satisfy $S_{i}, \quad i=$ $1, \ldots, 4-S_{1}=\mathrm{SP}, \mathrm{S}_{2}=\mathrm{NS}, \mathrm{S}_{3}=\mathrm{WSP}, \mathrm{S}_{4}=\mathrm{WNS}-$ according to whether $\tau_{i}=1$ of $\tau_{l}=0$. For example, the usual $l_{1}$-norm is of type 0011 , that is, $l_{1}$ does not have the SP and not NS but it does have the WSP and WNS.

Only six types are allowed by the implications $\mathrm{SP} \Rightarrow \mathrm{NS} \Rightarrow$ WNS and $\mathrm{SP} \Rightarrow \mathrm{WSP} \Rightarrow$ WNS: 1111, 0111, 0101, 0011, 0001, 0000. For reflexive spaces, the types 0111, 0011 and 0001 are excluded, For Schur-spaces, the types 0101, 0001 and 0000 are excluded.

In Theorem 2, $c_{0}$ is considered as real or complex Banach space.

THEOREM 2. RENORMINGS OF $c_{0}$. Given any of the six possible types 1111, 0111, 0101, 0011, 0001 or 0000, there is an equivalent norm on $c_{0}$ which is of this type. In particular, for $\vartheta= \pm 1$ and $0<\alpha \leq 1$, put 
$\varepsilon_{i}=\alpha 3^{-i}$, define $y_{l, j} \in l_{1}, 1 \leq i<j, y_{j} \in l_{1}$ and $y \in l_{1}$ by

$$
\begin{aligned}
& y_{i, j}(\nu)= \begin{cases}0, & j<\nu \text { or } i=\nu, \\
1-\varepsilon_{i}, & j=\nu, \\
2 \varepsilon_{\nu}, & j>\nu \neq i,\end{cases} \\
& y_{j}(\nu)=\left\{\begin{array}{ll}
0, & j<\nu, \\
1-\varepsilon_{\nu}, & j=\nu, \\
\vartheta \varepsilon_{\nu}, & j>\nu,
\end{array} \quad y(\nu)=\varepsilon_{\nu}\right.
\end{aligned}
$$

and consider the following norms on $c_{0}$ :

$$
\begin{gathered}
\|x\|_{\alpha}=\sup \left\{\left|\left\langle y_{i, j}, x\right\rangle\right| \mid 1 \leq i<j\right\}, \quad\|x\|_{\vartheta, \alpha}=\sup _{j \in \mathbf{N}}\left|\left\langle y_{j}, x\right\rangle\right|, \\
\|x\|_{\vartheta, \alpha}^{\prime}=\max \left\{\|x\|_{\alpha},\|x\|_{\vartheta, \alpha}\right\}, \quad\|x\|_{\vartheta, \alpha}^{\prime \prime}=\max \left\{\|x\|_{\vartheta, \alpha}^{\prime},\|x\|_{5}\right\}, \\
\|x\|_{5}=\left\{\sum_{\nu=1}^{\infty} 2^{-\nu} \frac{|x(\nu)|^{2}}{36}+\frac{1}{4} \sup \left\{\left|\sum_{\nu \in F} x(\nu)\right|^{2}|F \subset \mathbf{N},| F \mid \leq 5\right\}\right\}^{1 / 2} .
\end{gathered}
$$

Then, all these norms are equivalent to \|\|$_{\infty}$ and we have

(1) (a) $\left\|e_{n}-e_{k}\right\|_{\alpha} \leq 1-2 \varepsilon_{k}$.

(b) $\left\{u_{n}\right\}=\left\{\sum_{\nu=1}^{n}\left(e_{\nu+1}-3^{-v} e_{1}\right)\right\}$ is \|\|$_{\alpha}$-limit constant with $\Lambda_{\alpha}\left(u_{n}\right)=1$.

(c) $\left\{v_{n}\right\}=\left\{u_{n}+e_{n+2}\right\}$ is \|\|$_{\alpha}$-limit-affine with $\Lambda_{\alpha}\left(v_{n}\right)=1-$ $2 \varepsilon_{n+2}$.

(d) If $\left\{x_{n}\right\}$ is \|\|$_{\alpha}$-limit-affine, $x_{n} \rightarrow x \in l_{\infty}$ pointwise and $\Lambda_{\alpha}\left(x_{n}\right) \rightarrow 1$, then there exist increasing sequences $\left\{j_{n}\right\}$ and $\left\{m_{n}\right\}$ such that $x\left(j_{n}\right) \rightarrow \beta \rho$ and $x_{m_{n}}\left(j_{n}\right) \rightarrow \beta,|\beta|=1$, $\operatorname{Re} \rho \geq 1 / 2$.

(2) (a) $\left\{e_{n}\right\}$ is \|\|$_{\vartheta, \alpha}$-limit-affine with $\Lambda_{\vartheta \alpha}\left(e_{n}\right)=1-\vartheta \varepsilon_{n}$.

(b) $\left\{u_{n}\right\}$ is \|\|$_{+1, \alpha}$-limit constant with $\Lambda_{+1, \alpha}\left(u_{n}\right)=1$.

(c) $\left\{v_{n}^{\prime}\right\}=\left\{u_{n} / 6+e_{n+2}\right\}$ is \|\|$_{+1, \alpha}$-limit-affine with $\Lambda_{+1, \alpha}\left(v_{n}^{\prime}\right)=1-\varepsilon_{n+2}$.

(d) If $\left\{x_{n}\right\}$ is \|\|$_{\vartheta, \alpha^{-}}$-limit-affine, $x_{n} \rightarrow x \in l_{\infty}$ pointwise, $\Lambda_{\vartheta, \alpha}\left(x_{n}\right) \rightarrow 1, \vartheta\left(1-\Lambda_{\vartheta, \alpha}\left(x_{n}\right)\right) \leq 0$ and $\Lambda_{\alpha}\left(x_{n}\right)$ exists for all $n$ then $\Lambda_{a}\left(x_{n}\right) \geq \max \left\{1, \Lambda_{\vartheta, \alpha}\left(x_{n}\right)\right\}$ for all $n$.

(3) (a) $\left\|e_{n}-e_{k}\right\|_{5} \leq 2 / 3 \leq 1-\varepsilon_{k}$.

(b) $\left\|v_{n}^{\prime}-v_{k}^{\prime}\right\|_{5} \leq \sqrt{26} / 6 \leq 1-\varepsilon_{k+2}$.

(c) Every \|\|$_{5}$-limit-affine sequence is one-dimensional.

(d) If $x_{n} \rightarrow x \in l_{\infty}$ pointwise and $x\left(j_{n}\right) \rightarrow \beta \rho$ and $x_{n}\left(j_{n}\right) \rightarrow \beta$ for some increasing sequence $\left\{j_{n}\right\}$, and if $|\beta|=1, \operatorname{Re} \rho \geq$ $1 / 2$, then $\lim \sup _{n \rightarrow \infty}\left\|x_{n}-x_{k}\right\|_{5} \geq 3 / 2$ for all $k$. 
(4) \|\|$_{\alpha}$ is of type 0011.

(5) (a) $\left\{e_{n}\right\}$ is \|\|$_{+1, \alpha}^{\prime}$-limit-affine with $\Lambda_{+1, \alpha}^{\prime}\left(e_{n}\right)=1-\varepsilon_{n}$.

(b) $\left\{u_{n}\right\}$ is \|\|$_{+1, \alpha}^{\prime}$-limit constant with $\Lambda_{+1, \alpha}^{\prime}\left(u_{n}\right)=1$.

(c) \|\|$_{+1, \alpha}^{\prime}$ is of type 0001 .

(6) (a) $\left\{e_{n}\right\}$ is \|\|$_{+1, \alpha}^{\prime \prime}$-limit-affine with $\Lambda_{+1, \alpha}^{\prime \prime}\left(e_{n}\right)=1-\varepsilon_{n}$.

(b) \|\|$_{+1, \alpha}^{\prime \prime}$ is of type 0101.

(7) (a) $\left\{e_{n}\right\}$ is \|\|$_{-1, \alpha}^{\prime \prime}$-limit-affine with $\Lambda_{-1, \alpha}^{\prime \prime}\left(e_{n}\right)=1+\varepsilon_{n}$.

(b) \|\|$_{-1, \alpha}^{\prime \prime}$ is of type 1111.

(8) Consider the norm $\|x\|_{\alpha}^{\prime \prime \prime}=\left\{\frac{1}{2}\|x\|_{\alpha / 2}^{2}+\frac{1}{2}\|V x\|_{+1, \alpha}^{\prime \prime 2}\right\}^{1 / 2}$, $V x(i)=x(i+2)-x(i+3)$.

(a) $\left\{v_{n}\right\}$ is \|\|$_{\alpha}^{\prime \prime \prime}$-limit-affine with $\Lambda_{\alpha}^{\prime \prime \prime}\left(v_{n}\right)=1-\varepsilon_{n+2}$.

(b) \|\|$_{\alpha}^{\prime \prime \prime}$ is of type 0111.

(9) The original norm \|\|$_{\infty}$ is of type 0000.

Proof. The proof of (1a, b, c), (2a, b, c), (3a, b, c), (5a, b), (6a), (7a), (8a) and (9) is straightforward and omitted. For (3d), observe that

$$
\begin{aligned}
\left\|x_{n}-x_{k}\right\|_{5} & \geq \frac{1}{2}\left|x_{n}\left(j_{n}\right)-x_{k}\left(j_{n}\right)+\sum_{\nu=1}^{4}\left(x_{n}\left(j_{m+\nu}\right)-x_{k}\left(j_{m+\nu}\right)\right)\right| \\
\underset{n \rightarrow \infty}{\rightarrow} & \frac{1}{2}\left|\beta+\sum_{\nu=1}^{4}\left(x\left(j_{m+\nu}\right)-x_{k}\left(j_{m+\nu}\right)\right)\right| \\
& \rightarrow \underset{m \rightarrow \infty}{ } \frac{1}{2}|\beta+4 \beta \rho| \geq \frac{1}{2}(4 \operatorname{Re} \rho+1) \geq \frac{3}{2} .
\end{aligned}
$$

Assume first (1d) and (2d) to be already proved.

Using the methods described in [10], the following implications are immediate:

$(1) \Rightarrow(4),((1 d) \&(2 d)) \&((5 a) \&(5 b)) \Rightarrow(5 c)$,

$((1 \mathrm{~d}) \&(2 \mathrm{~d}) \&(3 \mathrm{c}, \mathrm{d})) \&(6 \mathrm{a}) \Rightarrow(6 \mathrm{~b}),(1 \mathrm{~d}) \&(2 \mathrm{~d}) \&(3 \mathrm{c}, \mathrm{d}) \Rightarrow(7 \mathrm{~b})$,

(6b) $\&(4) \&(8 a) \Rightarrow(8 b)$.

So, it remains to show (1d) and (2d).

Proof of (1d). Assume that $\left\{x_{n}\right\}$ is \|\|$_{\alpha}$-limit-affine, $x_{n} \rightarrow x \in l_{\infty}$ pointwise - i.e., weak* in $l_{\infty}$ - and

(i) $\Lambda_{n}:=\Lambda_{\alpha}\left(x_{n}\right) \rightarrow 1$.

Passing to a subsequence and applying Proposition 2, we find $1 \leq i_{n}<j_{n}$ and $\gamma, \beta \in \mathbf{C},|\beta|=1$, such that

(ii) $\left\langle y_{i_{n}, j_{n}}, x_{n}\right\rangle \rightarrow \gamma,\left\langle y_{i_{n}, j_{n}}, x_{k}\right\rangle \rightarrow \gamma-\Lambda_{k} \beta$.

Putting $\eta=\gamma \beta^{*}, \tilde{x}_{n}=\beta^{*} x_{n}$ and $\tilde{x}=\beta^{*} x$, we arrive at

(iii) $\left\langle y_{i_{n}, j_{n}}, \tilde{x}_{n}\right\rangle \rightarrow \eta$.

(iv) $\left\langle y_{i_{n}, J_{n}}, \tilde{x}_{k}\right\rangle \rightarrow \eta-\Lambda_{k}$. 
We know from (v) of Proposition 2 that $i_{n} \rightarrow \infty$ and $j_{n} \rightarrow \infty$. Switching to a subsequence, we may assume that $\xi=\lim _{n \rightarrow \infty} \tilde{x}_{n}\left(j_{n}\right)$ and $\rho=\lim _{n \rightarrow \infty} \tilde{x}\left(j_{n}\right)$ both exist. Then

$$
\begin{aligned}
& \text { (iii) } \Rightarrow \eta=2\langle y, \tilde{x}\rangle+\xi \text { and } \\
& \text { (iv) } \Rightarrow \eta-1 \underset{k \rightarrow \infty}{\leftarrow} \eta-\Lambda_{k}=2\left\langle y, \tilde{x}_{k}\right\rangle \underset{k \rightarrow \infty}{\rightarrow} 2\langle y, \tilde{x}\rangle .
\end{aligned}
$$

Thus $\xi=1$ and $2\left\langle\stackrel{k}{\langle}, \tilde{x}-\tilde{x}_{k}\right\rangle=\Lambda_{k}-1$. Finally, we obtain

$$
\begin{aligned}
\Lambda_{k} & \leftarrow\left\|\tilde{x}_{n}-\tilde{x}_{k}\right\|_{\alpha} \geq\left|\left\langle y_{J_{k}, J_{n}}, \tilde{x}_{n}-\tilde{x}_{k}\right\rangle\right| \\
& \rightarrow\left|\Lambda_{k}-1-2 \varepsilon_{J_{k}}\left(\tilde{x}-\tilde{x}_{k}\right)\left(j_{k}\right)+1-\varepsilon_{j_{k}}\right| \\
& =\left|\Lambda_{k}+2 \varepsilon_{j_{k}}\left(\tilde{x}_{k}\left(j_{k}\right)-\tilde{x}\left(j_{k}\right)-1 / 2\right)\right| \\
& \geq \Lambda_{k}+2 \varepsilon_{j_{k}}\left(\operatorname{Re} \tilde{x}_{k}\left(j_{k}\right)-\operatorname{Re} \tilde{x}\left(j_{k}\right)-1 / 2\right) .
\end{aligned}
$$

Hence,

$$
\operatorname{Re} \rho \underset{k \rightarrow \infty}{\leftarrow} \operatorname{Re} \tilde{x}\left(j_{k}\right) \geq \operatorname{Re} \tilde{x}_{k}\left(j_{k}\right)-1 / 2 \underset{k \rightarrow \infty}{\rightarrow} 1 / 2
$$

Proof of (2d). Assume that $\left\{x_{n}\right\}$ is \|\|$_{\vartheta, \alpha}$-limit-affine, $x_{n} \rightarrow x \in$ $l_{\infty}$ pointwise, $\Lambda_{n}^{\prime}=\Lambda_{\vartheta, \alpha}\left(x_{n}\right) \rightarrow 1, \quad \vartheta\left(1-\Lambda_{n}^{\prime}\right) \leq 0$ and $\Lambda_{k}=$ $\lim _{n \rightarrow \infty}\left\|x_{n}-x_{k}\right\|_{\alpha}$ exists for all $k$.

Using essentially the same method as in the proof of (1d) we may assume that $\left\langle y, \tilde{x}-\tilde{x}_{k}\right\rangle=\Lambda_{k}^{\prime}-1, \tilde{x}_{n}\left(j_{n}\right) \rightarrow 1$ and $\tilde{x}\left(j_{n}\right) \rightarrow \rho$ for some increasing sequence $\left\{j_{n}\right\}$ where $\tilde{x}_{n}=\beta^{*} x_{n}$ and $\tilde{x}=\beta^{*} x$ for some $\beta \in \mathbf{C}$ with $|\beta|=1$. Hence, we have

$$
\begin{gathered}
\Lambda_{k} \underset{n \rightarrow \infty}{\leftarrow}\left\|x_{n}-x_{k}\right\|_{\alpha} \geq\left|\left\langle y_{j_{n}-1, J_{n}}, \tilde{x}_{n}-\tilde{x}_{k}\right\rangle\right| \underset{n \rightarrow \infty}{\rightarrow} 1+2 \vartheta\left(\Lambda_{k}^{\prime}-1\right) \\
= \begin{cases}\Lambda_{k}^{\prime}+\left(\Lambda_{k}^{\prime}-1\right) \geq \Lambda_{k}^{\prime} \geq 1, & \vartheta=1, \\
1+2\left(1-\Lambda_{k}^{\prime}\right) \geq 1 \geq \Lambda_{k}^{\prime}, & \vartheta=-1 .\end{cases}
\end{gathered}
$$

For the main statement of Theorem 2 only the case $\vartheta=+1$ and $\alpha=1$ is needed. The norm \|\|$_{-1, \alpha}^{\prime \prime}$ and the parameter $\alpha$ are introduced in view of Theorem 4, the complete solution of P1.

In the next Theorem 3, the main statement of Theorem 2 is extended from $c_{0}$ to arbitrary infinite dimensional separable normed spaces; of course, we have to pay regard to the type-restrictions for reflexive and Schur spaces.

Theorem 3. Renormings of SEPARABLE InfINITE DIMENSIONAL SPACES. Let $X$ be an infinite dimensional separable normed space. Let $\tau_{1} \tau_{2} \tau_{3} \tau_{4}$ be any type which is compatible with the topological structure of 
$X: \tau_{1} \tau_{2} \tau_{3} \tau_{4}=1111,0111,0101,0011,0001$ or 0000 if $X$ is non-Schur, nonreflexive, $\tau_{1} \tau_{2} \tau_{3} \tau_{4}=1111,0101$ or 0000 if $X$ is reflexive, $\tau_{1} \tau_{2} \tau_{3} \tau_{4}=$ 1111, 0111 or 0011 if $X$ is Schur.

Then, $X$ admits an equivalent norm which is of type $\tau_{1} \tau_{2} \tau_{3} \tau_{4}$. Especially, given $\vartheta= \pm 1$ and $0<\alpha \leq 1$, there is a sequence $\left\{x_{n}\right\} \subset X$ and an equivalent norm \|\|$_{\vartheta, \alpha}$ on $X$ such that $\left\{x_{n}\right\}$ is \|\|$_{\vartheta, \alpha}$-limit-affine with $\Lambda_{\vartheta, \alpha}\left(x_{n}\right)=1-\vartheta \alpha 3^{-n}$ and $\left(X,\|\|_{\vartheta, \alpha}\right)$ has $N S$ (the SP if $\left.\vartheta=-1\right)$; if $X$ is non-Schur, then $\left\{x_{n}\right\}$ can be chosen to converge weakly.

For the proof of Theorem 3, we need some elementary facts which are also useful in other applications. In Lemmas $1-4, X$ is assumed to be a separable infinite dimensional normed space.

LEMMA 1. If $\left\{x_{n}\right\} \subset X$ is bounded and has no Cauchy subsequence, then there is a subsequence $\left\{x_{m_{n}}\right\}$ and a bounded sequence $\left\{x_{n}^{*}\right\} \subset X^{*}$ which converges to 0 in $\sigma\left(X^{*}, X\right)$ and is biorthogonal to $\left\{x_{m_{n}}\right\}$, that is $\left\langle x_{n}^{*}, x_{m_{k}}\right\rangle=\delta_{n, k}$.

Proof. By the lemma of [9], there is a bounded sequence $\left\{y_{n}^{*}\right\}$ in $X^{*}$ and a subsequence $\left\{x_{m_{n}}\right\}$ such that

$$
\left\langle y_{n}^{*}, x_{m_{k}}-x_{m_{l}}\right\rangle=\delta_{k, n}-\delta_{l, n} .
$$

Passing to a subsequence, we may assume that $y_{n}^{*} \rightarrow y^{*} \in X^{*}$ in $\sigma\left(X^{*}, X\right)$. Using (i) we obtain $\left\langle y^{*}, x_{m_{k}}-x_{m_{l}}\right\rangle=0$. If we call $y_{n}^{*}-y^{*}$ again $y_{n}^{*}$, we additionally have $y_{n}^{*} \rightarrow 0$ in $\sigma\left(X^{*}, X\right)$. Formula (i) implies that $\left\langle y_{n}^{*}, x_{m_{k}}\right\rangle=\alpha_{n}$ for all $k \neq n$, where $\alpha_{n} \rightarrow 0$. If $\alpha_{n}=0$ for all $n$, we put $x_{n}^{*}=y_{n}^{*}$. If not, we choose $N$ with $\alpha_{N} \neq 0$, put $x_{n}^{*}=y_{N+n}^{*}-$ $\alpha_{N+n} \alpha_{N}^{-1} y_{N}^{*}$ and denote $m_{N+n}$ again by $m_{n}$.

Lemma 2. If $\left\{w_{n}^{*}\right\} \subset W^{*}, W \subset X$, is bounded and converges to 0 in $\sigma\left(W^{*}, W\right)$, then there are extensions $y_{n}^{*} \in X^{*}$ of $w_{n}^{*}$ such that $\left\{y_{n}^{*}\right\}$ is bounded and converges to 0 in $\sigma\left(X^{*}, X\right)$.

Proof. Choose extensions $x_{n}^{*} \in X^{*}$ of $w_{n}^{*}$ with $\left\|x_{n}^{*}\right\|=\left\|w_{n}^{*}\right\| \leq K$. Let $d$ be a translation invariant metric on $X^{*}$ which induces $\sigma\left(X^{*}, X\right)$ on $B=\left\{x^{*} \in X^{*} \mid\left\|x^{*}\right\| \leq K\right\}$. Since every $\sigma\left(X^{*}, X\right)$-cluster point of $\left\{x_{n}^{*}\right\}$ belongs to $B \cap W^{\perp}$, we know that $d\left(x_{n}^{*}, B \cap W^{\perp}\right) \rightarrow 0$, i.e., there is a sequence $\left\{z_{n}^{*}\right\} \subset B \cap W^{\perp}$ with $d\left(x_{n}^{*}, z_{n}^{*}\right) \rightarrow 0$. Put $y_{n}^{*}=x_{n}^{*}-z_{n}^{*}$.

LEMMA 3. If $\left\{x_{n}\right\} \subset X$ is bounded and has no weakly convergent subsequence, then there is a bounded sequence $\left\{y_{n}^{*}\right\} \subset X^{*}$ which converges 
to 0 in $\sigma\left(X^{*}, X\right)$ and a subsequence $\left\{x_{m_{n}}\right\}$ such that

$$
\left\langle y_{n}^{*}, x_{m_{k}}\right\rangle= \begin{cases}1, & k \geq n, \\ 0, & k<n .\end{cases}
$$

Proof. By Lemma 1, there is a subsequence $\left\{x_{m_{n}}\right\}$ and a bounded sequence $\left\{x_{n}^{*}\right\} \subset X^{*}$ with $\left\langle x_{n}^{*}, x_{m_{k}}\right\rangle=\delta_{n, k}$ and $x_{n}^{*} \rightarrow 0$ in $\sigma\left(X^{*}, X\right)$. We further may assume that $\left\langle x^{*}, x_{m_{n}}\right\rangle \rightarrow 1$ for some $x^{*} \in X^{*}$, even that $\sum_{n=1}^{\infty}\left|\left\langle x^{*}, x_{m_{n}}\right\rangle-1\right|<\infty$. Putting $z^{*}=x^{*}-\sum_{n=1}^{\infty}\left(\left\langle x^{*}, x_{m_{n}}\right\rangle-1\right) x_{n}^{*}$, we obtain $\left\langle z^{*}, x_{m_{n}}\right\rangle=1$ for all $n$. So, for $M=\left\|z^{*}\right\|$, we have

$$
\left|\sum_{i=1}^{k} \lambda_{i}\right|=\left|\left\langle z^{*}, \sum_{i=1}^{k} \lambda_{i} x_{m_{t}}\right\rangle\right| \leq M\left\|\sum_{i=1}^{k} \lambda_{i} x_{m_{t}}\right\|
$$

for all $\lambda_{i}, i=1, \ldots, k, k \in \mathbf{N}$.

Let $u_{n}^{*}$ be the restriction of $\sum_{i=1}^{n} x_{i}^{*}$ to the span of $\left\{x_{m_{1}}\right\}_{i=1}^{n}$. Formula (i) implies $\left\|u_{n}^{*}\right\| \leq M$. Let $v_{n}^{*} \in W^{*}, W$ the closed span of $\left\{x_{m_{n}}\right\}_{n=1}^{\infty}$, be extensions of $u_{n}^{*}$ with $\left\|v_{n}^{*}\right\| \leq M$. Put $w_{1}^{*}=\left.z^{*}\right|_{W}$ and $w_{n}^{*}=w_{1}^{*}-v_{n-1}^{*}$, $n \geq 2$. Then $\left\{w_{n}^{*}\right\}$ is bounded, $w_{n}^{*} \rightarrow 0$ in $\sigma\left(W^{*}, W\right)$ and

$$
\left\langle w_{n}^{*}, x_{m_{k}}\right\rangle= \begin{cases}1, & k \geq n, \\ 0, & k<n,\end{cases}
$$

Application of Lemma 2 concludes the proof.

Lemma 4. If $\left\{x_{n}\right\} \subset X$ and $\left\{x_{n}^{*}\right\} \subset X^{*}$ are bounded such that $\left\langle x_{n}^{*}, x_{m}\right\rangle \rightarrow 0$ for $n \rightarrow \infty$ and fixed $m$ as well as for $m \rightarrow \infty$ and fixed $n$, then, given $\delta>0$, there are increasing sequences $\left\{m_{n}\right\}$ and $\left\{m_{n}^{\prime}\right\}$ such that $\left|\left\langle x_{m_{n}}^{*}, x_{m_{k}^{\prime}}\right\rangle\right| \leq \delta$ for all $n, k$.

Proof. Put $p_{1}=1$ and choose inductively $p_{n+1}$ so that $\left|\left\langle x_{p_{n}}^{*}, x_{k}\right\rangle\right| \leq \delta$ for all $k \geq p_{n+1}$. We obtain $\left|\left\langle x_{p_{n}}^{*}, x_{p_{k}}\right\rangle\right| \leq \delta$ if $k>n$. The same argument applied to $\left\{x_{p_{n}}^{*}\right\}$ and $\left\{x_{p_{n}}\right\}$ instead of $\left\{x_{n}\right\}$ and $\left\{x_{n}^{*}\right\}$ yields a subsequence $\left\{p_{n}^{\prime}\right\}$ of $\left\{p_{n}\right\}$ with $\left|\left\langle x_{p_{n}^{\prime}}^{*}, x_{p_{k}^{\prime}}\right\rangle\right| \leq \delta$ for all $n \neq k$. Put $m_{n}=p_{2 n-1}^{\prime}$ and $m_{n}^{\prime}=p_{2 n}^{\prime}$.

Proof of Theorem 3. (1) Pick $z_{n}^{*} \in X^{*}$ with $\left\|z_{n}^{*}\right\|=1$ and $\|x\|=$ $\sup _{n \in \mathrm{N}}\left|\left\langle z_{n}^{*}, x\right\rangle\right|$ for all $x \in X$. Put

$$
\|x\|^{\prime}=\left\{\|x\|^{2}+\sum_{n=1}^{\infty} 2^{-n}\left|\left\langle z_{n}^{*}, x\right\rangle\right|^{2}\right\}^{1 / 2} .
$$

Then, every \|\|$^{\prime}$-limit-affine sequence in $X$ is one-dimensional, i.e. \|\|$^{\prime}$ is of type 1111 . 
(2) According to Lemma 1, choose bounded biorthogonal sequences $\left\{x_{n}\right\}$ and $\left\{x_{n}^{*}\right\}$ such that $x_{n}^{*} \rightarrow 0$ in $\sigma\left(X^{*}, X\right)$ and, if $X$ is non-Schur, $x_{n} \rightarrow 0$ weakly. Define $T: X \rightarrow c_{0}$ by $(T x)(i)=\left\langle x_{l}^{*}, x\right\rangle$. Put $d=$ $\sup _{n, m}\left\|x_{n}-x_{m}\right\|^{\prime}$ and $\|x\|^{\prime \prime}=\max \left\{d^{-1}\|x\|^{\prime},\|T x\|_{\infty}\right\}$. Then, $\left\{x_{n}\right\}$ is \|\|$^{\prime \prime}$-limit-constant with $\Lambda^{\prime \prime}\left(x_{n}\right)=1$ and \|\|$^{\prime \prime}$ is of type 0000, if $X$ is non-Schur, and of type 0011, if $X$ is Schur.

Put

$$
\|x\|_{\vartheta, \alpha}=\max \left\{\frac{2}{3} d^{-1}\|x\|^{\prime},\|T x\|_{\vartheta, \alpha}^{\prime \prime}\right\} .
$$

Then, $\left\{x_{n}\right\}$ is \|\|$_{\vartheta, \alpha}$-limit-affine with $\Lambda_{\vartheta, \alpha}\left(x_{n}\right)=1-\vartheta \alpha 3^{-n},\|\|_{-1, \alpha}$ is of type 1111 , and \|\|$_{+1, \alpha}$ is of type 0101 , if $X$ is non-Schur, and of type 0111 , if $X$ is Schur.

(3) Assume $X$ to be non-reflexive non-Schur. According to Lemma 3, there are bounded sequences $\left\{x_{n}\right\}$ and $\left\{y_{n}^{*}\right\}$ such that $y_{n}^{*} \rightarrow 0$ in $\sigma\left(X^{*}, X\right)$ and

$$
\left\langle y_{n}^{*}, x_{m}\right\rangle= \begin{cases}1, & m \geq n \\ 0, & m<n .\end{cases}
$$

Define $T: X \rightarrow c_{0}$ by $(T x)(i)=\left\langle y_{l}^{*}, x\right\rangle$. Consider

$$
y_{n}=\sum_{\nu=1}^{n} 3^{-\nu} x_{1}+x_{n+1}-x_{1} .
$$

Put $d_{0}=\sup _{n, m}\left\|y_{n}-y_{m}\right\|^{\prime}$ and $\|x\|^{\prime \prime \prime}=\max \left\{d_{0}^{-1}\|x\|^{\prime},\|T x\|_{1}\right\}$. Since $T y_{n}$ $=u_{n},\left\{y_{n}\right\}$ is \|\|$^{\prime \prime \prime}$-limit-constant and \|\|$^{\prime \prime \prime}$ is of type 0011 .

Consider now

$$
y_{n}^{\prime}=\sum_{\nu=1}^{n+1} 3^{-\nu} x_{2}+x_{2 n+3}-x_{2}+6\left(x_{2 n+4}-x_{2 n+3}\right) .
$$

Put $d_{1}=\sup \left\|y_{n}^{\prime}-y_{m}^{\prime}\right\|^{\prime}$ and $\|x\|_{1}=\max \left\{\frac{2}{3} d_{1}^{-1}\|x\|^{\prime},\|T x\|_{1}^{\prime}\right\}$. Since $T y_{n}^{\prime}=$ $v_{n}\left\{y_{n}^{\prime}\right\}$ is \|\|$_{1}$-limit-affine with $\Lambda_{1}\left(y_{n}^{\prime}\right)=1-3^{-n-2}$ and \|\|$_{1}$ is of type 0111 .

Assume that $X$ has no subspace isomorphic to $l_{1}$. We may then additionally assume that $\left\{x_{n}\right\}$ is weak Cauchy. Put $y_{1}^{\prime \prime}=x_{1}$ and $y_{n}^{\prime \prime}=x_{n}$ $-x_{n-1}, \quad n \geq 2$. Then $T y_{n}^{\prime \prime}=e_{n}$ and $y_{n}^{\prime \prime} \rightarrow 0$ weakly. Set $d_{2}=$ $\max \left\{d_{0}, \sup _{n, m}\left\|y_{n}^{\prime \prime}-y_{m}^{\prime \prime}\right\|^{\prime}\right\}$ and $\|x\|_{2}=\max \left\{\frac{2}{3} d_{2}^{-1}\|x\|^{\prime},\|T x\|_{+1,1}^{\prime}\right\}$. Then, $\left\{y_{n}^{\prime \prime}\right\}$ is \|\|$_{2}$-limit-affine with $\Lambda_{2}\left(y_{n}^{\prime \prime}\right)=1-3^{-n},\left\{y_{n}\right\}$ is \|\|$_{2}$-limit-constant with $\Lambda_{2}\left(y_{n}\right)=1$ and \|\|$_{2}$ is of type 0001 .

Finally, assume $X$ to have a subspace isomorphic to $l_{1}$. Let $\left\{w_{n}\right\} \subset X$ and $\left\{w_{n}^{*}\right\} \subset X^{*}$ be bounded and biorthogonal such that $w_{n} \rightarrow 0$ weakly and $w_{n}^{*} \rightarrow 0$ in $\sigma\left(X^{*}, X\right)$. Choose $\left\{x_{n}^{\prime}\right\} \subset X$ equivalent to the unit vector 
basis of $l_{1}$. Passing to a subsequence of $\left\{x_{n}^{\prime}\right\}$ we may assume that $\left\{\left\langle w_{k}^{*}, x_{n}^{\prime}\right\rangle\right\}$ is Cauchy for all $k$. So, some suitable subsequence $\left\{x_{n}\right\}$ of $\left\{x_{n+1}^{\prime}-x_{n}^{\prime}\right\}$ satisfies $\left\langle w_{k}^{*}, x_{n}\right\rangle \rightarrow 0$ for all $k$. Since $\left\{x_{n}\right\}$ can be chosen to be equivalent to the unit vector basis of $l_{1}$, there is a bounded sequence $\left\{y_{n}^{*}\right\}$ in $X^{*}$ which converges to 0 in $\sigma\left(X^{*}, X\right)$ and satisfies

$$
\left\langle y_{n}^{*}, x_{m}\right\rangle= \begin{cases}1, & m \geq n \\ 0, & m<n .\end{cases}
$$

Using Lemma 4, we may assume that $\left\langle y_{n}^{*}, w_{k}\right\rangle \mid \leq \delta^{2} / 2$ and $\left|\left\langle w_{n}^{*}, x_{k}\right\rangle\right| \leq$ $\delta^{2} / 3$ for all $k, n$, where $\delta$ is chosen so that $\delta\|x\|_{\infty} \leq\|x\|_{+1,1}^{\prime} \leq 1 / \delta\|x\|_{\infty}$ for all $x \in c_{0}$. Define $T,\left\{y_{n}\right\}$ and $d_{0}$ as above and $T^{\prime}: X \rightarrow c_{0}$ by $\left(T^{\prime} x\right)(i)=\left\langle w_{i}^{*}, x\right\rangle$. Put $d_{3}=\max \left\{d_{0}, \sup _{n, m}\left\|w_{n}-w_{m}\right\|^{\prime}\right\}$ and $\|x\|_{3}=$ $\max \left\{\frac{2}{3} / d_{3}\|x\|^{\prime},\|T x\|_{+1,1}^{\prime},\left\|T^{\prime} x\right\|_{+1,1}^{\prime}\right\}$. Then, we have $T^{\prime} w_{n}=e_{n}$. Moreover, for $n>k$ :

$$
\begin{aligned}
\left\|T\left(w_{n}-w_{k}\right)\right\|_{+1,1}^{\prime} & \leq\left\|T\left(w_{n}-w_{k}\right)\right\|_{\infty} / \delta \leq \delta \\
& =\delta\left\|T^{\prime}\left(w_{n}-w_{k}\right)\right\|_{\infty} \leq\left\|T^{\prime}\left(w_{n}-w_{k}\right)\right\|_{+1,1}^{\prime}
\end{aligned}
$$

and

$$
\begin{aligned}
\left\|T^{\prime}\left(y_{n}-y_{k}\right)\right\|_{+1,1}^{\prime} & \leq\left\|T^{\prime}\left(y_{n}-y_{k}\right)\right\|_{\infty} / \delta \leq \delta \\
& =\delta\left\|T\left(y_{n}-y_{k}\right)\right\|_{\infty} \leq\left\|T\left(y_{n}-y_{k}\right)\right\|_{+1,1}^{\prime} .
\end{aligned}
$$

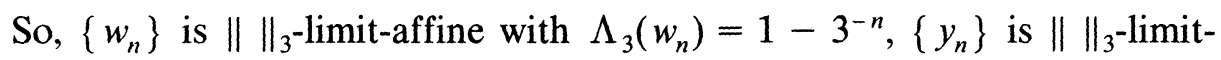
constant with $\Lambda_{3}\left(y_{n}\right)=1$ and \|\|$_{3}$ is of type 0001 .

TheOREM 4. Solution of P1. Let || be a symmetric norm on $\mathbf{R}^{N}$; put $Z=\left(\mathbf{R}^{N},||\right)$. Then $(W) N S$ is preserved under the Z-direct-sum-operation - that is, the $Z$-direct sum of $X_{1}, \ldots, X_{N}$ has $(W) N S$ whenever all $X_{\imath}$ have (W) NS - if and only if || satisfies (SC).

In particular, if || does not satisfy (SC) and if separable infinite dimensional normed (non-Schur) spaces $X_{1} \ldots X_{N}$ are given, then there exist equivalent norms \|\|$_{i}^{\prime}$ on $X_{i}$ such that $Y_{i}=\left(X_{i},\|\|_{i}^{\prime}\right)$ has NS but $\left(\sum_{1=1}^{N} \oplus Y_{i}\right)_{Z}$ does not have $(W) N S$; moreover, at least one of the \|\|$_{i}^{\prime}$ can be chosen so that $Y$, has the SP.

Proof. Sufficiency is proved in [10]. We show necessity. Let || not satisfy (SC) and let separable infinite dimensional normed (non-Schur) spaces $X_{1}, \ldots, X_{N}$ be given. There are $\xi_{i} \geq 0, \vartheta_{\imath}= \pm 1$ and $0<\alpha_{i} \leq 1, i=1, \ldots, N$, such that the line segment joining $\left(\xi_{i}\right)_{i=1}^{N}$ and $\left(\xi_{i}\left(1-\vartheta_{i} \alpha_{i} / 3\right)\right)_{i=1}^{N}$ belongs to the unit sphere of $Z$. Then, according to Theorem 3 , there exist (weakly convergent) sequences $\left\{x_{n}(i)\right\}_{n=1}^{\infty}$ 
in $X_{i}$ and equivalent norms \|\|$_{i}^{\prime}=\|\|_{\vartheta_{i}, \alpha_{i}}$ on $X_{i}$ such that $Y_{i}=\left(X_{l},\|\|_{i}^{\prime}\right)$ has NS or even the SP if $\vartheta_{i}=-1$ - such an $i$ must exist - and $\left\{x_{n}(i)\right\}$ is \|\|$_{i}^{\prime}$-limit-affine with $\Lambda_{i}^{\prime}\left(x_{n}(i)\right)=1-\vartheta_{\imath} \alpha_{l} 3^{-n}$. Obviously, $\left\{\left(\xi_{l} x_{n}(i)\right)_{i=1}^{N}\right\}$ is limit-constant in $\left(\sum_{l=1}^{N} \oplus Y_{i}\right)_{Z}$.

5. Isonormal structure. In this last section, we prove that $c_{0}(I)$ has (W)INS if and only if the index set $I$ is countable. Using a result of Troyanski [13], we characterize (W)INS of spaces with a symmetric basis. This characterization fails if the basis is only unconditional. First, we need some preliminaries. For completeness, we give the definition of the space $c_{0}(I)$ : Given a set $I, c_{0}(I)$ is the Banach space of all functions $x$ from $I$ to $\mathbf{R}$ or $\mathbf{C}$ which vanish at infinity (i.e., for all $\varepsilon>0$ there is a finite subset $J$ of $I$ such that $|x(i)|<\varepsilon$ for all $i \in I \backslash J)$ endowed with the sup-norm \|\|$_{\infty}$. Given an element $x$ of $c_{0}(I)$, the support of $x$ is the set

$$
\operatorname{supp}(x)=\{i \mid x(i) \neq 0\} \text {. }
$$

Let $H(I)$ be the set of all $\eta \in c_{0}(I)$ with finite support and values in $\{-1,0,1\}$.

A sequence $\left\{x_{n}\right\}$ in a normed space $X$ is said to be asymptotically isometric to the unit vector basis $\left\{e_{n}\right\}$ of $c_{0}$ if $T e_{n}=x_{n}$ extends to an isomorphism of $c_{0}$ into $X$ with $\|T\| \leq 1$ and

$$
\liminf _{n \rightarrow \infty}\left\{\|T \xi\| \mid \xi \in c_{0}, \operatorname{supp}(\xi) \subset\{i \mid i>n\},\|\xi\|_{\infty}=1\right\}=1 \text {. }
$$

Proposition 3. Let $Y$ be a subset of a normed space $X$ such that

$$
\left\|\sum_{y \in Y} \eta(y) y\right\| \leq 1 \quad \text { for all } \eta \in H(Y) .
$$

Then, for all $\xi \in c_{0}(Y)$, the sum $T_{Y} \xi=\sum_{y \in Y} \xi(y) y$ converges unconditionally and defines an operator of $c_{0}(Y)$ into $X$ with

$$
\begin{aligned}
& 2 \inf _{y \in Y}\|y\|-1 \leq\left\|T_{Y}\right\| \leq 1 \quad \text { and } \\
& \left\|T_{Y} \xi\right\| \geq 2 \sup _{y \in Y}|\xi(y)|\|y\|-\|\xi\|_{\infty} .
\end{aligned}
$$

If, additionally, $Y=\left\{y_{n} \mid n \in \mathbf{N}\right\}$ with $\lim _{n \rightarrow \infty}\left\|y_{n}\right\|=1$, then, for some $m \in \mathbf{N},\left\{y_{m+n}\right\}$ is asymptotically isometric to the unit vector basis of $c_{0}$.

Proof. (1) By induction on the number of different elements of $R(\xi)=\{|\xi(y)| \mid y \in \operatorname{supp}(\xi)\}$ we show that $\left\|T_{Y} \xi\right\| \leq\|\xi\|_{\infty}$ for all $\xi \in$ $c_{0}(Y)$ with finite support. Hypothesis (i) implies the induction basis. For the induction conclusion, fix $\xi \in c_{0}(Y)$ with finite support and $r_{1}=$ $\min R(\xi)<r_{2}=\max R(\xi)$. Put $Z=\left\{y \in Y|| \xi(Y) \mid=r_{1}\right\}$ and define 
$\xi_{\nu} \in c_{0}(Y)$ by $\xi_{\nu}(y)=\xi(y), y \in Y \backslash Z, \nu=1,2$, and $\xi_{1}(z)=0, \xi_{2}(z)=$ $\left(r_{2} / r_{1}\right) \xi(z), z \in Z$. Then $R\left(\xi_{\nu}\right)=R(\xi) \backslash\left\{r_{1}\right\}$ and $\xi=(1-\lambda) \xi_{1}+\lambda \xi_{2}$, $\lambda=r_{1} / r_{2}$. The induction hypothesis applied to $\xi_{\nu}, \nu=1,2$, yields

$$
\left\|T_{Y} \xi\right\| \leq(1-\lambda)\left\|T_{Y} \xi_{1}\right\|+\lambda\left\|T_{Y} \xi_{2}\right\| \leq(1-\lambda) r_{2}+\lambda r_{2}=r_{2}=\|\xi\|_{\infty} .
$$

The induction is complete.

(2) By (1), the sum $T_{Y} \xi=\sum_{y \in Y} \xi(y) y$ converges unconditionally for all $\xi \in c_{0}(Y)$ and, hence, defines an operator with $\left\|T_{Y}\right\| \leq 1$.

(3) Fix $\xi \in c_{0}(Y)$ and $y_{0} \in Y$. Then

$$
\begin{aligned}
\left\|T_{y} \xi\right\| & \geq 2\left|\xi\left(y_{0}\right)\right|\left\|y_{0}\right\|-\left\|\xi\left(y_{0}\right) y_{0}-\sum_{y \neq y_{0}} \xi(y) y\right\| \\
& \geq 2\left|\xi\left(y_{0}\right)\right|\left\|y_{0}\right\|-\|\xi\|_{\infty} .
\end{aligned}
$$

Taking the supremum over $y_{0} \in Y$ yields (ii).

(4) If $Y=\left\{y_{n}\right\},\left\|y_{n}\right\| \rightarrow 1$, then pick $m$ with $\inf _{n>m}\left\|y_{n}\right\| \geq 3 / 4$. Putting $Y^{\prime}=\left\{y_{n}\right\}_{n>m}$, we obtain from (ii) that $1 / 2 \leq\left\|T_{Y^{\prime}}\right\| \leq 1$ and $\left\|T_{Y^{\prime}} \xi\right\| \geq 2 \inf _{n>N}\left\|y_{n}\right\|-1 \rightarrow 1$ if $\|\xi\|_{\infty}=1$ and $\operatorname{supp}(\xi) \subset\{n \mid n>N\}$, $N \rightarrow \infty$.

Proposition 4. If the normed space $X$ contains a sequence $\left\{x_{n}\right\}$ asymptotically isometric to the unit vector basis of $c_{0}$, then $X$ does not have WNS.

Proof. The sequence $\left\{x_{n}\right\}$ converges weakly to 0 and $\left\|x_{n}-x_{k}\right\| \leq 1$. Moreover, $\left\|x_{n}-\sum_{k=1}^{m} \lambda_{k} x_{k}\right\| \geq 2\left\|x_{n}\right\|-1 \rightarrow 1$ if $m<n \rightarrow \infty$ and $0 \leq$ $\lambda_{h} \leq 1$.

Corollary 2. If the normed space $X$ contains a sequence $\left\{x_{n}\right\}$ with $\left\|x_{n}\right\| \rightarrow r$ and $\left\|\sum_{n=1}^{\infty} \eta(n) x_{n}\right\| \leq r$ for all $\eta \in H(\mathbf{N})$ for some $r>0$, then $X$ does not have WNS.

THEOREM 5. The space $c_{0}(I)$ has (W)INS if and only if I is countable.

Proof. Sufficiency is shown in [10]. We prove necessity. Let \|\| be an equivalent norm on $c_{0}(I), I$ uncountable. An easy application of Day's well known construction [2] yields:

(*) Given a countable subset $C$ of $I$, there is a sequence $Y=\left\{y_{n}\right\}$ in $c_{0}(I)$ such that

(i) $\left\|y_{n}\right\|_{\infty}=1$ and $\operatorname{supp}\left(y_{n}\right) \subset \operatorname{supp}\left(y_{n+1}\right) \subset I \backslash C$ for all $n$.

(ii) $r(Y)=\lim _{n \rightarrow \infty}\left\|y_{n}\right\|$ exists and $\|z\| \leq r(Y)$ for all $z \in c_{0}(I)$ with $\|z\|_{\infty} \leq 1$ and $\operatorname{supp}(z) \subset I \backslash C \backslash \bigcup_{n=1}^{\infty} \operatorname{supp}\left(y_{n}\right)$. 
Starting with $C_{1}=\varnothing$ and using transfinite induction over all ordinals $\gamma<\omega_{1}, \omega_{1}$ the first uncountable ordinal, we obtain by application of $(*)$ a transfinite sequence $\left\{Y_{\gamma}\right\}_{\gamma<\omega_{1}}$ of sequences $Y_{\gamma}=\left\{y_{\gamma, n}\right\}_{n=1}^{\infty}$ with (i) and (ii) for $C=C_{\gamma}=\bigcup_{\alpha<\gamma} \bigcup_{n=1}^{\infty} \operatorname{supp}\left(y_{\alpha, n}\right)$. Since, by construction, $\left\{r\left(Y_{\gamma}\right)\right\}_{\gamma<\omega_{1}}$ is non-increasing, there must be an ordinal $\gamma_{0}<\omega_{1}$ such that $r\left(Y_{\gamma}\right)=r$ for all $\gamma \geq \gamma_{0}$ and some $r>0$. So, for all $n$, there are $k_{n}$ such that $\left\|x_{n}\right\|>r-1 / n$ for $x_{n}=y_{\gamma_{0}+n, k_{n}}$. Given $\eta \in H(\mathbf{N})$, we have $\left\|\sum_{n=1}^{\infty} \eta(n) x_{n}\right\|_{\infty} \leq 1$ and $\operatorname{supp}\left(\sum_{n=1}^{\infty} \eta(n) x_{n}\right) \subset I \backslash C_{\gamma_{0}+1}$, so $\left\|\sum_{n=1}^{\infty} \eta(n) x_{n}\right\|$ $\leq r\left(Y_{\gamma_{0}}\right)=r$. Corollary 2 yields the conclusion.

We combine Theorem 5 with a result of Troyanski which characterizes Banach spaces with a symmetric basis that can be equivalently renormed to be uniformly convex in every direction (cf. [13]). Here, an unconditional (not necessarily countable) basis is called symmetric if all sequences of elements of the basis are mutually equivalent basic sequences.

Corollary 3. Let $X$ be a Banach space with a symmetric basis. Then, the following are equivalent.

(1) $X$ is isomorphic to a Banach space with the $S P$.

(2) $X$ is isomorphic to a Banach space with the WSP.

(3) X has INS

(4) X has WINS.

(5) $X$ is isomorphic to a Banach space which is uniformly convex in every direction.

(6) $X$ is not isomorphic to $c_{0}(I)$ with uncountable $I$.

Proof. The implications (1) $\Rightarrow(2) \Rightarrow(4)$ and (1) $\Rightarrow(3) \Rightarrow(4)$ are trivial. $(5) \Rightarrow(1)$ : See $[10$, Appendix]. (4) $\Rightarrow(6)$ : Theorem 5. (6) $\Rightarrow(5)$ : See [13].

The implication (4) $\Rightarrow(5)$ in Corollary 3 can not be extended to the wider class of Banach spaces with an unconditional basis (even when restricted to reflexive spaces) as the following example shows.

EXAMPLE. A reflexive Banach space $X$ with a monotone unconditional basis such that neither $X$ nor $X^{*}$ can be renormed to be uniformly convex in every direction but such that $X$ and $X^{*}$ (in the dual norm) both have the SP:

An unconditional basis $\left\{e_{i}\right\}_{l \in I}$ is monotone if the projections

$$
P_{f}: \sum_{i \in I} \alpha_{i} e_{t} \mapsto \sum_{j \in f} \alpha_{j} e_{j}, \quad f \subset I \text { finite }
$$


all have norm 1. In [7], the following is introduced: Let $I$ be an uncountable index set and $\Phi$ a family of finite non-empty subsets of $I$ with $\{i\} \in \Phi$ for all $i \in I$ and $f \in \Phi$ if $f \neq \varnothing, f \subset \varphi$ and $\varphi \in \Phi$. Let $\mathscr{F}$ be the set of all collections $F$ of finitely many mutually disjoint elements of $\Phi$. Define

$$
\begin{aligned}
\lambda_{12} & =\lambda_{12}(\Phi)=\left\{x: I \rightarrow \mathbf{R} \mid \sup _{F \in \mathscr{F}}\|x\|_{F}<\infty\right\}, \\
\|x\|_{F} & =\left[\sum_{\varphi \in F}\left(\sum_{i \in \varphi}|x(i)|\right)^{2}\right]^{1 / 2}, \quad F \in \mathscr{F}, \\
\|x\|_{12} & =\sup _{F \in \mathscr{F}}\|x\|_{F}, \quad x \in \lambda_{12} .
\end{aligned}
$$

Then, $\left(\lambda_{12},\|\|_{12}\right)$ is a Banach space with monotone unconditional boundedly complete basis $\left\{e_{i}\right\}_{\in I}, e_{i}(i)=1, e_{l}(j)=0, j \neq i$. Since, obviously, $\|x\|_{2}=\left(\sum_{i \in I}|x(i)|^{2}\right)^{1 / 2} \leq\|x\|_{12},\|x\|=\left(\|x\|_{2}^{2}+\|x\|_{12}^{2}\right)^{1 / 2}$ is an equivalent norm on $\lambda_{12}$ with $\|x\|_{2} \leq\|x\| / \sqrt{2}$ with respect to which $\lambda_{12}$ has the SP.

There is given an example of $I$ and $\Phi$ in [7] such that $\lambda_{12}$ is reflexive and $\lambda_{12} \oplus \lambda_{12}^{*}$ as well as $\left(\lambda_{12} \oplus \lambda_{12}^{*}\right)^{*}$ cannot be renormed to be uniformly convex in every direction. This set $\Phi$ has the property that, given arbitrary different $i, j \in I$, there are only finitely many $\varphi \in \Phi$ containing both $i$ and $j$. So, given any finite subset $f \subset I$, the set

$$
f^{\prime}=\bigcup\{\varphi \in \Phi|| f \cap \varphi \mid \geq 2\} \cup f
$$

is finite. For this $I$ and $\Phi$, let $X$ be the space $\lambda_{12} \oplus \lambda_{12}^{*}$ with the norm $\left(\|x\|^{2}+\left\|x^{*}\right\|^{* 2}\right)^{1 / 2}$, where \|\|$^{*}$ is the norm on $\lambda_{12}^{*}$ dual to \|\| . So, neither $X$ nor $X^{*}$ can be renormed to be uniformly convex in every direction. It remains to show that both $X$ and $X^{*}$ have the SP. It suffices to establish the SP of $\left(\lambda_{12}^{*},\|\|^{*}\right)$. For this purpose, we only have to verify $(*)$ in Proposition 5 below for $c=\sqrt{2+\sqrt{2}}$ and $f^{\prime}$ as above. To this end, let $x$, $y \in \lambda_{12}$ be given with $\|x\|=\|y\|=1$. Fix $F \in \mathscr{F}$ and a finite subset $f$ of $I$. Put $\quad F_{1}=\left\{\varphi \in F \mid \varphi \subset f^{\prime}\right\}, \quad F_{2}=\{\varphi \in F \mid \varphi \cap f=\varnothing\}, \quad F_{3}=$ $F \backslash F_{1} \backslash F_{2}$. For all $\varphi \in F_{3}$ there is an $i_{\varphi} \in I$ such that $\varphi \cap f=\left\{i_{\varphi}\right\}$. Put $\tilde{x}=P_{f} x, \tilde{y}=y-P_{f^{\prime}} y$. Then, $\|\tilde{x}\| \leq 1,\|\tilde{y}\| \leq 1$ and $\|\tilde{x}\|_{2} \leq 1 / \sqrt{2}$. So,

$$
\begin{aligned}
\|\tilde{x}+\tilde{y}\|_{F}^{2}= & \|\tilde{x}\|_{F_{1}}^{2}+\|\tilde{y}\|_{F_{2}}^{2}+\|\tilde{x}\|_{F_{3}}^{2}+\|\tilde{y}\|_{F_{3}}^{2} \\
& +2 \sum_{\varphi \in F_{3}}\left|\tilde{x}\left(i_{\varphi}\right)\right|\left(\sum_{i \in \varphi}|\tilde{y}(i)|\right) \\
\leq & \|\tilde{x}\|_{12}^{2}+\|\tilde{y}\|_{12}^{2}+2\|\tilde{x}\|_{2}\|\tilde{y}\|_{F_{3}} .
\end{aligned}
$$


Taking the supremum over $F \in \mathscr{F}$ we obtain as desired

$$
\begin{aligned}
\|\tilde{x}+\tilde{y}\|^{2} & =\|\tilde{x}\|_{2}^{2}+\|\tilde{y}\|_{2}^{2}+\|\tilde{x}+\tilde{y}\|_{12}^{2} \\
& \leq\|\tilde{x}\|_{2}^{2}+\|\tilde{x}\|_{12}^{2}+\|\tilde{y}\|_{2}^{2}+\|\tilde{y}\|_{12}^{2}+2\|\tilde{x}\|_{2}\|\tilde{y}\| \leq 2+\sqrt{2} .
\end{aligned}
$$

Proposition 5. Let $X$ have an unconditional basis $\left\{e_{1}\right\}_{i \in I}$ with (*) There is a $c<2$ such that, for each finite subset $f$ of $I$, there is a finite $f^{\prime} \subset I$ with $f \subset f^{\prime}$ such that

$$
\left\|P_{f} x+y-P_{f^{\prime}} y\right\| \leq c \quad \text { whenever }\|x\|=\|y\|=1 .
$$

If $X$ is reflexive, then $X^{*}$ has the $\mathrm{SP}$.

Proof. Let $P_{f}^{*}$ be the dual projection. If $X^{*}$ does not have the SP, then there is a sequence $\left\{x_{n}^{*}\right\}$ in $X^{*}$ such that

$$
x_{n}^{*} \rightarrow 0 \text { weakly, }\left\|x_{n}^{*}\right\| \rightarrow 1 \text { and } \Lambda\left(x_{n}^{*}\right) \rightarrow 1 .
$$

Fix $\varepsilon>0$ with $2-8 \varepsilon>c(1+2 \varepsilon)$. Choose $k$ such that $\Lambda\left(x_{k}^{*}\right)<1+\varepsilon$ and $\left\|x_{n}^{*}\right\|>1-\varepsilon$ for all $n \geq k$. Pick a finite $f \subset I$ such that

$$
\left\|x_{k}^{*}-P_{f}^{*} x_{k}^{*}\right\|<\varepsilon \text { for all finite } \tilde{f} \supset f .
$$

Take $n>k$ so that $\left\|P_{\tilde{f}}^{*} x_{n}^{*}\right\|<\varepsilon$ for all $\tilde{f} \subset f^{\prime}$ and $\left\|x_{n}^{*}-x_{k}^{*}\right\|<$ $\Lambda\left(x_{k}^{*}\right)+\varepsilon$. Then

$\left\|P_{f}^{*}\left(x_{n}^{*}-x_{k}^{*}\right)\right\|>1-4 \varepsilon$ and $\left\|x_{n}^{*}-x_{k}^{*}-P_{f^{\prime}}^{*}\left(x_{n}^{*}-x_{k}^{*}\right)\right\|>1-4 \varepsilon$.

So, there are $x, y \in X$ such that $\|x\|=\|y\|=1$ and

$$
\begin{aligned}
& \left\langle x, P_{f}^{*}\left(x_{n}^{*}-x_{k}^{*}\right)\right\rangle>1-4 \varepsilon \text { and } \\
& \left\langle y, x_{n}^{*}-x_{k}^{*}-P_{f^{\prime}}^{*}\left(x_{n}^{*}-x_{k}^{*}\right)\right\rangle>1-4 \varepsilon .
\end{aligned}
$$

So,

$$
\begin{aligned}
c(1+2 \varepsilon) & >\left\|P_{f} x+y-P_{f^{\prime}} y\right\|\left(\Lambda\left(x_{k}^{*}\right)+\varepsilon\right) \\
& >\left\|P_{f} x+y-P_{f^{\prime}} y\right\|\left\|x_{n}^{*}-x_{k}^{*}\right\| \\
& \geq\left\langle P_{f} x+y-P_{f^{\prime}} y, x_{n}^{*}-x_{k}^{*}\right\rangle>2-8 \varepsilon .
\end{aligned}
$$

contradicting the choice of $\varepsilon$.

\section{REFERENCES}

[1] M. S. Brodskii and D. P. Mil'man, On the center of a convex set, Dokl. Akad. Nauk SSSR, 59 (1948), 837-840 (Russian).

[2] M. M. Day, Strict Convexity and smoothness, Trans. Amer. Math. Soc., 78 (1955), 516-528. 
[3] W. A. Kirk, A fixed point theorem for mappings which do not increase distances, Amer. Math. Monthly, 72 (1965), 1004-1006.

[4] _ Fixed Point Theory for Nonexpansive mappings, Proc. Workshop on Fixed Point Theory, Univ. de Sherbrooke, 1980; Lecture Notes in Math., Springer (1981), 4484-505.

[5] _ Nonexpansive mappings and normal structure in Banach spaces, Proc. Research Workshop on Banach space Theory, Univ. of Iowa, 1981.

[6] _ Fixed point theory for nonexpansive mappings II.

[7] D. N. Kutzarova and S. L. Troyanski, Reflexive Banach spaces without equivalent norms which are uniformly convex or uniformly differentiable in every direction, Stud. Math., 72 (1982), 91-95.

[8] T. R. Landes, Das Iterationstheorem und Fixpunktsätze bei normaler Struktur, Dissertation, Paderborn 1981.

[9] _ A characterization of totally normal structure, Arch. Math., 37 (1981), 248-255.

[10] , Permanence properties of normal structure, Pacific J. Math., 10 (1984), 125-143.

[11] - Normal structure and weakly normal structure of Orlicz sequence spaces, Trans. Amer. Math. Soc., 285 (1984), 523-534.

[12] _ Normal structure and weakly normal structure of modular sequence spaces, preprint.

[13] S. L. Troyanski, On nonseparable Banach space with a symmetric basis, Stud. Math., 53 (1975), 253-263.

Received October 10, 1984 and in revised form February 20, 1985.

UNIVERSITY OF PADERBORN

POSTFACH 1621

D-4790 PADERBORN

WEST GERMANY 



\section{PACIFIC JOURNAL OF MATHEMATICS EDITORS}

V. S. VARADARAJAN

(Managing Editor)

University of California

Los Angeles, CA 90024

HERBERT CLEMENS

University of Utah

Salt Lake City, UT 84112

R. FINN

Stanford University

Stanford, CA 94305
HERMANN FLASCHKA

University of Arizona

Tucson, AZ 85721

RAMESH A. GANGOLLI

University of Washington

Seattle, WA 98195

VAUGHAN F. R. JONES

University of California

Berkeley, CA 94720

ROBION KIRBY

University of California

Berkeley, CA 94720
C. C. MOORE

University of California

Berkeley, CA 94720

H. SAMELSON

Stanford University

Stanford, CA 94305

HAROLD STARK

University of California, San Diego

La Jolla, CA 92093

\section{ASSOCIATE EDITORS}

R. ARENS

E. F. BECKENBACH

B. H. NEUMANN (1906-1982)
F. WOLF

K. YOSHIDA

\section{SUPPORTING INSTITUTIONS}

UNIVERSITY OF ARIZONA

UNIVERSITY OF BRITISH COLUMBIA

CALIFORNIA INSTITUTE OF TECHNOLOGY

UNIVERSITY OF CALIFORNIA

MONTANA STATE UNIVERSITY

UNIVERSITY OF NEVADA, RENO

NEW MEXICO STATE UNIVERSITY

OREGON STATE UNIVERSITY
UNIVERSITY OF OREGON UNIVERSITY OF SOUTHERN CALIFORNIA

STANFORD UNIVERSITY

UNIVERSITY OF HAWAII

UNIVERSITY OF TOKYO

UNIVERSITY OF UTAH

WASHINGTON STATE UNIVERSITY

UNIVERSITY OF WASHINGTON 


\section{Pacific Journal of Mathematics}

\section{Vol. 123, No. $1 \quad$ March, 1986}

Maria Emilia Alonso García, A note on orderings on algebraic varieties $\ldots \ldots 1$

F. S. De Blasi and Józef Myjak, On continuous approximations for

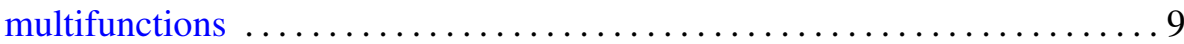

Frank Albert Farris, An intrinsic construction of Fefferman's CR metric . . . 33 Antonio Giambruno, P. Misso and Francisco César Polcino Milies, Derivations with invertible values in rings with involution $\ldots . \ldots . \ldots .47$

Dan Haran and Moshe Jarden, The absolute Galois group of a pseudo real

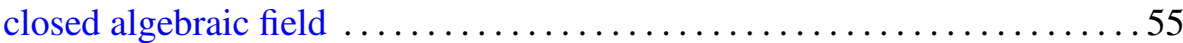

Telemachos E. Hatziafratis, Integral representation formulas on analytic

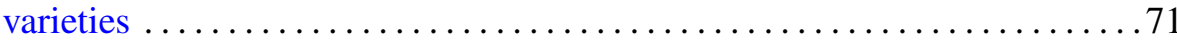

Douglas Austin Hensley, Dirichlet's theorem for the ring of polynomials

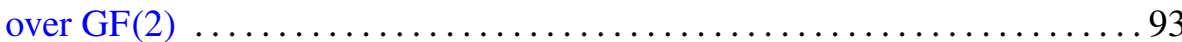

Sofia Kalpazidou, On a problem of Gauss-Kuzmin type for continued

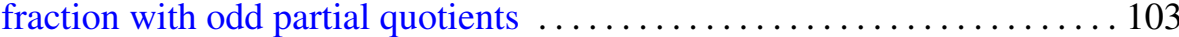

Harvey Bayard Keynes and Mahesh Nerurkar, Ergodicity in affine

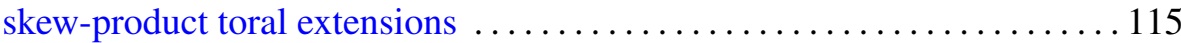

Thomas Landes, Normal structure and the sum-property $\ldots \ldots \ldots \ldots \ldots 127$

Anthony To-Ming Lau and Viktor Losert, Weak*-closed complemented invariant subspaces of $L_{\infty}(G)$ and amenable locally compact groups ...149 Andrew Lelek, Continua of constant distances in span theory . . . . . . . . 161 Dominikus Noll, Sums and products of $B_{r}$ spaces $\ldots \ldots \ldots \ldots \ldots \ldots \ldots \ldots$ Lucimar Nova, Fixed point theorems for some discontinuous operators 189

A. A. S. Perera and Donald Rayl Wilken, On extreme points and support points of the family of starlike functions of order $\alpha$

Massimo A. Picardello, Positive definite functions and $L^{p}$ convolution operators on amalgams ........................

Friedrich Roesler, Squarefree integers in nonlinear sequences ......... 223

Theodore Shifrin, The osculatory behavior of surfaces in $\mathbf{P}^{5}$ 